

\section{Leveraging Digital Infrastructure for Data Analysis: An Example in Bariatric Surgery}

John Hagen, Lazar Klein, Ethan Miller and Shirley Solomon

\section{Abstract}

Background: The digitalization of healthcare information provides hospitals with the ability to gain insight into patterns and associations pertaining to disease and management. Using bariatric patient data as an example provided an opportunity to explore the potential of electronic medical record (EMR) data to generate insights.

Objective: The aim of this study was to extract EMR data pertaining to bariatric patient information as a means to explore predictive factors of weight loss post-bariatric surgery.

Methods: We conducted a retrospective cohort study of patients undergoing bariatric surgery between January 1 , 2018, and April 30, 2019, at Humber River Hospital. Multiple linear regression was used to examine whether age, pre-surgery body mass index (BMI), comorbidities and mental health disorders predicted higher weight loss 6 months following bariatric surgery.

Results: A total of 502 patients were included in the final analysis. Age ( $\beta=0.04[95 \% \mathrm{Cl} 0.01,0.06], p=0.005)$, baseline BMI ( $ß=-0.16[95 \% \mathrm{Cl}-0.19,-0.13], p=<0.0001)$ and diabetes $(B=0.82[95 \% \mathrm{Cl} 0.23,1.42], p=0.007)$ were associated with weight loss six months post-bariatric surgery.

Conclusion: EMRs are a rich source of data with the potential to generate insights that can lead to improved care. 


\section{Introduction}

The advent of electronic medical records (EMRs) has allowed for extensive documentation and storage of critical patient data. EMRs provide access to information such as in-patient and outpatient records, laboratory results and imaging. In addition to recording and documenting patient information into EMRs, analysis of the data may be employed for clinical research and be used to guide clinical decisions. Healthcare institutions may extract patient data to gain insight into patterns and associations pertaining to disease and management. The aim of this study was to use EMR data to explore preoperative predictors of weight loss following bariatric surgery.

Obesity increases the risk of developing comorbidities such as diabetes, hypertension, hyperlipidemia and obstructive sleep apnea (CIHI 2014). Achieving significant weight loss post-bariatric surgery is key as it is correlated with success in improving existing comorbidities by reducing blood glucose or resolving hyperglycemia more effectively than medical therapy alone (Schauer et al. 2017). Several studies have found that comorbidities were important factors causing less excess weight loss following bariatric surgery (Carbonell et al. 2008; Júnior et al. 2011). This may pose a problem for patients with existing comorbidities, who may not lose as much excess weight after bariatric surgery as those without comorbidities.

In addition to physical comorbidities associated with obesity, psychological issues arise as well. In a study conducted by Goldsmith et al., (2006), it was found that $55.6 \%$ of obese patients $(n=54)$ met the criteria for major depression. In addition to the association between mental illness and obesity, mental illness might also play a role in preventing significant weight loss. A prospective analysis examining 104 bariatric surgery candidates found that patients with a current or lifetime history of mood disorders lost significantly less weight than patients without a psychiatric diagnosis following bariatric surgery (Semanscin-Doerr et al. 2010). Furthermore, a study conducted by Kinzl et al. (2006) exploring psychiatric disorders among bariatric surgery patients in relation to weight loss success found that there were poorer weight loss outcomes among patients with multiple psychiatric disorders.

EMRs contain a great deal of data that can be extracted and analyzed and allow for rapid and convenient data collection, which might later be used to examine data patterns and associations. With extensive data analysis, interventions might be discovered that improve patient care and management outcome. This study explored body mass index (BMI) reduction in bariatric patients using EMR-related variables from a single-site institution.

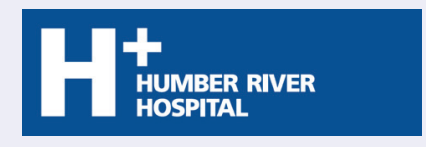

HUMBER RIVER HOSPITAL is one of Canada's largest community acute care hospitals, serving a population of more than 850,000 people in the northwest Greater Toronto Area. The multi-site hospital currently operates out of its Wilson Avenue acute care site and Finch and Church Street reactivation care centres with a total of 722 beds, just over 3,800 employees, approximately 700 physicians and over 1,000 volunteers.

Affiliated with the University of Toronto and Queen's University, Humber River Hospital is North America's first fully digital hospital. Part of Humber River Hospital's digital infrastructure includes completely automated laboratory services, robots sorting and mixing medications, electronic health records, tracking systems, for patients undergoing surgery, that update families through their cellphones and patient bedside computer terminals - all varieties of technologies that automate information, eliminate paper and provide a connected experience for patients, staff and families.

Humber River Hospital was awarded Accreditation with Exemplary Standing in 2018 and, since its opening in 2015, has received numerous awards and accolades for technological advancements and innovation (www.hrh.ca).

\section{Methods}

\section{Data source and data collection}

Data for this study were extracted from the Ontario Bariatric Registry (Anvari et al. 2019). The registry collects standardized information on consenting patients undergoing bariatric treatment at participating Bariatric Centres of Excellence in Ontario. A secondary analysis of de-identified data was conducted on patients undergoing bariatric surgery between January 1, 2018, and April 30, $2019(N=718)$, at Humber River Hospital (HRH). Data collected included baseline patient characteristics obtained at the initial assessment (age, height and weight), medical history (obesity-related comorbidities), history of mental health conditions (mood disorder, anxiety disorder), weight measurement on the day of surgery and 6-month followup assessment (weight at 6 months). The final dataset included 
only bariatric surgery patients who completed a baseline assessment as well as a 6-month follow-up assessment $(n=503 ; 70 \%$ of patients from the original dataset). Ethics clearance was granted by Veritas IRB, an external Research Ethics Board.

\section{Data analysis}

Variables at baseline were presented as frequency with percentage or mean with standard deviation. BMI was calculated as $\mathrm{kg} / \mathrm{m}^{2}$. The outcome variable was weight loss following bariatric surgery, which was calculated by subtracting the BMI on the day of surgery from the BMI measurement at 6 months following surgery for each patient. Predictor variables included age, baseline BMI (BMI on the day of surgery), obesity-related comorbidities in the past 12 months (diabetes type 1 and 2, hypertension, hyperlipidemia, chronic lung disease and sleep apnea) and a history of psychiatric illness (mood disorder and anxiety disorder).

The associations between independent variables and change in BMI 6 months following bariatric surgery were first evaluated using simple linear regression analyses. Next, multiple linear regression with backward elimination (probability for removal, $p>0.1$ ) was performed, with all independent variables entered simultaneously to examine the most significant factors associated with change in BMI at the 6-month follow-up.

All regression assumptions of linearity, normality of residuals and homoscedasticity of residuals were assessed and met. Collinearity diagnostics revealed that no multicollinearity was present in the final model. All analyses were conducted using SPSS Version 25 statistical software. A $p$-value $<0.05$ was considered statistically significant.

\section{Results}

Baseline variables are summarized in Table 1 . The average age of the sample was $44.4(S D=10.28)$ years, and the mean baseline $\mathrm{BMI}$ of the sample was $45.9(S D=7.6)$. Of the total sample, $83.7 \%$ reported having at least one obesity-related comorbidity in the past 12 months, including diabetes (21.7\%), hypertension (40.2\%), hyperlipidemia (25.8\%) and chronic lung disease (15.1\%). Additionally, 30.2\% reported having a history of mood disorder and $25.6 \%$ reported having a history of anxiety disorder.

Overall, study participants lost an average of $27.7 \mathrm{~kg}$ $(S D=8.7 \mathrm{~kg})$ from the day of surgery to the 6 -month followup. The mean 6-month postoperative change in BMI was -10.1 $(S D=3.0)$. The results from the simple linear regression analyses between the independent variables and postoperative weight loss are presented in Table 2. Younger age, higher baseline BMI, non-diabetic status, non-hypertension status and non-hyperlipidemia status were significantly associated with a greater reduction in BMI 6 months following bariatric surgery. In the final multiple regression analysis, only younger age, higher baseline $\mathrm{BMI}$ and non-diabetic status were associated with greater BMI reduction $\left(R^{2}=0.21, F(3,45.20), p<0.001\right)$. After controlling
TABLE 1.

Characteristics of study participants

\begin{tabular}{|c|c|}
\hline \multicolumn{2}{|c|}{ Mean (SD), frequency $(\%)(N=503)$} \\
\hline Age (yr) & $44.4(10.3)$ \\
\hline Baseline weight (kg) & $131.9(26.5)$ \\
\hline Baseline BMI (kg/m²) & $45.9(7.6)$ \\
\hline \multicolumn{2}{|c|}{ Obesity-related comorbidities (past 12 months) } \\
\hline Diabetes & $109(21.7 \%)$ \\
\hline Hypertension & $202(40.2 \%)$ \\
\hline Hyperlipidemia & $130(25.8 \%)$ \\
\hline Chronic lung disease & $76(15.1 \%)$ \\
\hline Sleep apnea & $328(65.2 \%)$ \\
\hline \multicolumn{2}{|c|}{ History of psychiatric illness } \\
\hline Mood disorder & $152(30.2 \%)$ \\
\hline Anxiety disorder & $129(25.6 \%)$ \\
\hline
\end{tabular}

for age, baseline weight and diabetes, non-hypertension status and non-hyperlipidemia status were not significantly associated with loss of body mass in the final model.

\section{Discussion}

In digital healthcare organizations such as $\mathrm{HRH}$, rich data are generated from multiple sources, including EMRs and administrative systems. Employing data analysis and data mining in healthcare can be a valuable technique for predicting various diseases and assisting physicians with clinical decisionmaking (Jothi and Husain 2015). This study analyzed EMR data pertaining to bariatric patient information as a means to explore potential associations between the data variables.

Obesity persists across the world as an epidemic disease contributing to the development of significant comorbidities, such as diabetes mellitus type 2, hypertension, coronary artery disease and cancer (Nickel et al. 2019). The onset of obesity varies greatly by age and ranges from childhood to adolescence and adulthood (Nickel et al. 2019). The findings from this study revealed that possible predictors of greater weight loss in BMI 6 months following bariatric surgery were younger age, higher baseline BMI and non-diabetic status. This model explained $21 \%$ of the variance in BMI loss. Our results are similar to those of some previous studies that identified non-diabetes status and younger age as possible predictors of better weight loss after surgery (Fox et al. 2015; Livhits et al. 2012; Ma et al. 2006; Mitchell et al. 2016; Nickel et al. 2019). Our study also identified a higher baseline BMI as a predictor of change in BMI; however, the findings in the literature on the association between preoperative BMI and weight loss following bariatric surgery were mixed 
TABLE 2.

Linear regression of BMI reduction six months following bariatric surgery and potential predictors

\begin{tabular}{|c|c|c|c|c|}
\hline \multirow{2}{*}{ Characteristic } & \multicolumn{2}{|c|}{ Simple regression } & \multicolumn{2}{|c|}{ Final multiple regression $(N=502)$} \\
\hline & B $(95 \% \mathrm{CI})$ & $p$-value & B (95\% CI) & $p$-value \\
\hline Age & 0.07 (0.04 to 0.09) & $<0.0001$ & $0.04(0.01$ to 0.06$)$ & 0.005 \\
\hline Baseline BMI & $-0.17(-0.20$ to -0.14$)$ & $<0.0001$ & $-0.16(-0.19$ to -0.13$)$ & $<0.0001$ \\
\hline \multicolumn{5}{|l|}{ Obesity-related comorbidities } \\
\hline Diabetes & $1.09(0.45$ to 1.73$)$ & 0.001 & 0.82 (0.23 to 1.42 ) & 0.007 \\
\hline Hypertension & $0.65(0.11$ to 1.19$)$ & 0.019 & & \\
\hline $\begin{array}{l}\text { Hyperlipidemia/high cholesterol/high } \\
\text { triglycerides }\end{array}$ & $1.16(0.56$ to 1.76$)$ & $<0.0001$ & & \\
\hline Chronic lung disease & 0.25 (-0.49 to 0.99$)$ & 0.508 & & \\
\hline Sleep apnea & $-0.22(-0.78$ to 0.34$)$ & 0.435 & & \\
\hline \multicolumn{5}{|l|}{ History of psychiatric illness } \\
\hline Mood disorder & $-0.07(-0.65$ to 0.51$)$ & 0.819 & & \\
\hline Anxiety disorder & $-0.39(-1.00$ to 0.22$)$ & 0.206 & & \\
\hline
\end{tabular}

(Livhits et al. 2012). These contradictory results could be due to the lack of a consistent metric for reporting weight loss outcome (i.e., change in BMI, total absolute weight loss, etc.).

Our study also found that a history of psychiatric illness, including mood and anxiety disorders, was not associated with greater weight loss in BMI 6 months following bariatric surgery. Our results are similar to those of previous studies that found no significant difference in the percentage of total weight loss following bariatric surgery across several psychiatric illnesses, including substance abuse and mood and anxiety disorders (Thomson et al. 2016). Our study supports other research suggesting that patients with psychiatric illness should not be excluded from bariatric surgery on the assumption that post-surgical weight loss will be poor and psychiatric symptoms following surgery will be worse (Thomson et al. 2016). This finding highlights the important issue of health inequity in accessing bariatric surgery. Jackson et al. (2014) suggested that the population that receives bariatric surgery does not reflect the individuals who need it the most. To move toward greater health equity in the treatment of obesity, further research that clarifies the predictors of successful bariatric surgery and closes the gaps in our understanding of the risks is necessary.

\section{Implications}

This study provided the opportunity to explore the potential of EMR data to generate actionable insights that foster improved care. Data analytics and data mining can support the ability of researchers and clinicians to improve the use of available research and evidence and capture care experience to create a continuous learning healthcare system (Lee and Yoon 2017). At $\mathrm{HRH}$, we are just beginning to access the massive repository of data that exists within our electronic world of healthcare. In building a data strategy, there is no standardized protocol to model or compare (Lee and Yoon 2017). Recognizing that the accumulation of big data is insufficient to solve problems or answer questions, $\mathrm{HRH}$ is developing a robust data analytics system with the goal of improving healthcare through predictive modelling for risk and resource use, disease and treatment heterogeneity, clinical decision support, quality of care and performance measurement.

\section{Limitations}

This study has some limitations, such as the low rate of follow-up data and the short follow-up time frame of 6 months; as such, the findings may be biased. Additional predictors affecting weight loss outcome, such as motivation to lose weight, dietary habits and levels of physical activity were not studied. Furthermore, the lack of an established metric for weight loss further limits comparability across studies (Adams et al. 2013). Although the most frequently reported outcome measure in bariatric literature is the percentage of excess weight loss (\%EWL), this measure may also not reflect "successful" weight loss as patients with a $\mathrm{BMI}$ in the higher ranges will often have a lower \%EWL than patients with a lower BMI despite achieving greater absolute weight loss (Adams et al. 2013).

\section{Conclusions}

EMRs are a rich source of data in healthcare organizations 
that can be extracted to gain insight into patterns and associations pertaining to disease and management. Obesity is a national epidemic that affects many Canadians. Interventions to combat obesity include lifestyle modification and bariatric surgery (CIHI 2014). By employing data analysis on bariatric patient data, we were able to explore the associations between data variables and provide insight into clinical practices that may support improved quality of care.

\section{What We Learned:}

1. Our example of data analytics generated actionable insights that may foster improved care in the bariatric patient population; for example, the focus on reducing childhood and adolescent obesity requires more than just therapies aimed at increasing healthy food consumption and exercise but may necessitate consideration of bariatric surgical intervention.

2. Similar to previous studies, our findings suggested that patients with psychiatric illness should not be excluded from bariatric surgery on the assumption that post-surgical weight loss will be poor and psychiatric symptoms following surgery will be worse.

3. The findings from our study highlight the important issue of health inequity in accessing bariatric surgery.

\section{Acknowledgements}

The authors would like to thank the Ontario Bariatric Network for providing access to the registry database and Ida Grisoni for her assistance in acquiring the data.

\section{References}

Adams, S.T., M. Salhab, Z.I. Hussain, G.V. Miller and S.H. Leveson. 2013. Roux-en-Y Gastric Bypass for Morbid Obesity: What Are the Preoperative Predictors of Weight Loss? Postgraduate Medical Journal 89(1053): 411-16. doi:10.1136/postgradmedj-2012-131310.

Anvari, M., A. Sharma, S. Yusuf, D. Hong, J.-E. Tarride, M. Tiboni et al. 2019. Bariatric Registry. Population Health Research Institute and the Centre for Surgical Invention and Innovation - Supported by the Ministry of Health and Long Term Care. Hamilton, ON: Authors.

Canadian Institute for Health Information (CIHI). 2014, May. Bariatric Surgery in Canada. Retrieved March 18, 2020. <https://secure.cihi.ca/ free_products/Bariatric_Surgery_in_Canada_EN.pdf $>$.

Carbonell, A.M., L.G. Wolfe, J.G., Meador, H.J. Sugerman, J.M. Kellum and J.W. Maher. 2008. Does Diabetes Affect Weight Loss after Gastric Bypass? Surgery for Obesity and Related Diseases 4(3): 441-44.

Fox, B., E. Chen, A. Suzo, S. Jolles, J.A., Greenberg, G.M. Campos et al. 2015. Dietary and Psych Predictors of Weight Loss after Gastric Bypass. Journal of Surgical Research 197(2): 283-90. doi:10.1016/j.jss.2015.04.019.

Goldsmith, S.J., K. Anger-Friedfeld, D. Rudolph, M. Boeck and L. Aronne. 2006. Psychiatric Illness in Patients Presenting for Obesity Treatment. International Journal of Eating Disorders 12(1): 63-71. doi:10.1159/000492000.
Jackson, T.D., R. Zhang, D. Glockler, J. Pennington, J.I. Reddigan, O.D. Rotstein et al. 2014. Health Inequity in Access to Bariatric Surgery: A Protocol for a Systematic Review. Systematic Reviews 3(1): 15. Jothi, N. and W. Husain. 2015. Data Mining in Healthcare - A Review. Procedia Computer Science 72: 306-13.

Júnior, W.S., J.L. do Amaral and C.B. Nonino-Borges. 2011. Factors Related to Weight Loss Up to 4 Years after Bariatric Surgery. Obesity Surgery 21(11): 1724-30.

Kinzl, J.F., M. Schrattenecker, C. Traweger, M. Mattesich, M. Fiala and W. Biebl. 2006. Psychosocial Predictors of Weight Loss after Bariatric Surgery. Obesity Surgery 16(12): 1609-14. doi:10.1381/096089206779319301.

Lee, C.H. and H.-J. Yoon. 2017. Medical Big Data: Promise and Challenges. Kidney Research and Clinical Practice 36(1): 3-11.

Livhits, M., C. Mercado, I. Yermilov, J.A. Parikh, E. Dutson, A. Mehran et al. 2012. Preoperative Predictors of Weight Loss following Bariatric Surgery: Systematic Review. Obesity Surgery 22(1): 70-89. doi:10.1007/s11695-011-0472-4.

Ma, Y., S.L. Pagoto, B.C. Olendzki, A.R. Hafner, R.A. Perugini, R. Mason et al. 2006. Predictors of Weight Status following Laparoscopic Gastric Bypass. Obesity Surgery 16(9): 1227-31.

Mitchell, J.E., N.J. Christian, D.R. Flum, A. Pomp, W.J. Pories, B.M. Wolfe et al. 2016. Postoperative Behavioral Variables and Weight Change 3 Years after Bariatric Surgery. JAMA Surgery 151(8): 752-57. doi:10.1001/jamasurg.2016.0395.

Nickel, F., J.R. de la Garza, F.S. Werthmann, L. Benner, C. Tapking, E. Karadza et al. 2019. Predictors of Risk and Success of Obesity Surgery. Obesity Facts 12(4): 427-39. doi:10.1159/000496939.

Schauer, P.R., D.L. Bhatt, J.P. Kirwan, K. Wolski, A. Aminian, S.A. Brethauer et al. 2017. Bariatric Surgery versus Intensive Medical Therapy for Diabetes - 5-Year Outcomes. New England Journal of Medicine 376(7): 641-51. doi:10.1056/NEJMoa1600869.

Semanscin-Doerr, D.A., A. Windover, K. Ashton and L.J. Heinberg. 2010. Mood Disorders in Laparoscopic Sleeve Gastrectomy Patients: Does It Affect Early Weight Loss? Surgery for Obesity and Related Diseases 6(2): 191-96. doi:10.1016/j.soard.2009.11.017.

Thomson, L., K.A. Sheehan, C. Meaney, S. Wnuk, R. Hawa and S. Sockalingam. 2016. Prospective Study of Psychiatric Illness as a Predictor of Weight Loss and Health Related Quality of Life One Year after Bariatric Surgery. Journal of Psychosomatic Research 86: 7-12. doi:10.1016/j.jpsychores.2016.04.008.

\section{About the Authors}

John Hagen, MD, FRCS(C), is a surgeon, Chief of Surgery, and Medical Director of Bariatrics at the Humber River Hospital, as well as a faculty member of the University of Toronto's MIS fellowship training program. He can be reached by email at JHagen@hrh.ca.

Lazar Klein, MD, MSc, $\mathrm{FRC}(\mathrm{C})$, is a surgeon at Humber River Hospital.

Ethan Miller, MD, MSc, BSc, is a research assistant at Humber River Hospital.

Shirley Solomon, MSc, BA, is a research coordinator at Humber River Hospital. 